\title{
Multimorbidity medications and poor asthma prognosis
}

\author{
Sébastien Chanoine ${ }^{1,2,3}$, Margaux Sanchez ${ }^{4,5}$, Isabelle Pin ${ }^{1,6}$, Sofia Temam ${ }^{4,5,7}$, \\ Nicole Le Moual ${ }^{4,5}$, Agnès Fournier ${ }^{8,9}$, Christophe Pison ${ }^{3,10,11}$, \\ Jean Bousquet ${ }^{4,5,12,13}$, Pierrick Bedouch $2,3,14$, Marie-Christine Boutron-Ruault ${ }^{8,9}$, \\ Raphaëlle Varraso ${ }^{4,5}$ and Valérie Siroux ${ }^{1}$
}

\begin{abstract}
Affiliations: ${ }^{1}$ Team of Environmental Epidemiology Applied to Reproduction and Respiratory Health, Institute for Advanced Biosciences, INSERM U1209, CNRS UMR 5309, Université Grenoble Alpes, Grenoble, France. ${ }^{2}$ Pôle Pharmacie, CHU Grenoble Alpes, Grenoble, France. ${ }^{3}$ Université Grenoble Alpes, Grenoble, France. 4INSERM U1168, VIMA, Aging and Chronic Diseases: Epidemiological and Public Health Approaches, Villejuif, France. ${ }^{5}$ Université Versailles St-Quentin-en-Yvelines, UMRS-S 1168, Montigny le Bretonneux, France. ${ }^{6}$ Clinique de Pédiatrie, Pôle Couple Enfant, CHU Grenoble Alpes, Grenoble, France. ${ }^{7}$ Université Paris-Sud, Faculté de Médecine, Le Kremlin-Bicêtre, France. ${ }^{8}$ INSERM, Centre for Research in Epidemiology and Population Health (CESP), UMRS 1018, Université Paris-Sud, UVSQ, Université Paris-Saclay, Villejuif, France. ${ }^{9}$ Institut Gustave Roussy, Villejuif, France. ${ }^{10}$ Service Hospitalier Universitaire Pneumologie-Physiologie, Pôle Thorax et Vaisseaux, CHU Grenoble Alpes, Grenoble, France. ${ }^{11}$ INSERM, Laboratoire de Bioénergétique Fondamentale et Appliquée, U1055, Grenoble, France. ${ }^{12}$ Clinique de Pneumologie, CHU de Montpellier, Montpellier, France. ${ }^{13} \mathrm{MACVIA-France,} \mathrm{Contre} \mathrm{les} \mathrm{Maladies} \mathrm{Chroniques} \mathrm{pour} \mathrm{un} \mathrm{Vleillissement} \mathrm{Actif} \mathrm{en}$ France, European Innovation Partnership on Active and Healthy Ageing Reference Site, Montpellier, France. ${ }^{14}$ CNRS, TIMC-IMAG UMR5525/ThEMAS, Université Grenoble Alpes, Grenoble, France.
\end{abstract}

Correspondence: Marie-Christine Boutron-Ruault, Centre for Research in Epidemiology and Population Health (CESP), UMRS 1018, Health Across Generation Team, 114 rue Edouard Vaillant, 94805 Villejuif, France. E-mail: marie-christine.boutronagustaveroussy.fr

@ERSpublications

Asthma phenotypes identified by multimorbidity medications are associated with asthma prognosis http://ow.ly/Phfq30iR1Qh

Cite this article as: Chanoine S, Sanchez M, Pin I, et al. Multimorbidity medications and poor asthma prognosis. Eur Respir J 2018; 51: 1702114 [https://doi.org/10.1183/13993003.02114-2017].

ABSTRACT Multimedication related to multimorbidity is common in the elderly with asthma. We aimed at comprehensively characterising medications used by elderly women and assessing how multimedication impacts on asthma prognosis.

We performed network-based analyses on drug administrative databases to visualise the prevalence of drug classes and their interconnections among 17458 elderly women from the Asthma-E3N study, including 4328 women with asthma. Asthma groups sharing similar medication profiles were identified by a clustering method relying on all medications and were studied in association with adverse asthma events (uncontrolled asthma, attacks/exacerbations and poor asthma-related quality of life).

The network-based analysis showed more multimedication in women with asthma than in those without asthma. The clustering method identified three multimedication profiles in asthma: "Few multimorbidity-related medications" (43.5\%), "Predominantly allergic multimorbidity-related medications" (32.8\%) and "Predominantly metabolic multimorbidity-related medications" (23.7\%). Compared with women belonging to the "Few multimorbidity-related medications" profile, women belonging to the two other profiles had an increased risk of uncontrolled asthma and asthma attacks/exacerbations, and had lower asthma-related quality of life.

The integrative data-driven approach on drug administrative databases identified specific multimorbidity-related medication profiles that were associated with poor asthma prognosis. These findings support the importance of multimorbidity in the unmet needs in asthma management. 


\section{Introduction}

Multimorbidity is a major problem in elderly adults (defined as $\geqslant 64$ years old), leading to multimedication $[1,2]$. It is common in asthma; older people with asthma had an average of $\mathrm{n}=8$ comorbidities versus $\mathrm{n}=3.4$ comorbidities for younger adults with asthma [3, 4]. The most common asthma-related multimorbidity includes allergic rhinitis, chronic sinusitis, sleep apnoea syndrome, lower airway diseases, particularly chronic obstructive pulmonary disease (COPD), gastro-oesophageal reflux disease (GORD), obesity, infections, hormonal disorders and psychological disorders [5]. Other chronic conditions have recently been suggested to be associated with asthma, such as diabetes mellitus, metabolic syndrome and cardiovascular diseases $[4,6]$. Multimorbidity in asthma may affect diagnosis, disease control/severity, lung function, health-related quality of life and asthma medication response, and therefore lead to increased medical costs [7].

Multimorbidity medications have been poorly addressed in asthma and never as a comprehensive assessment integrating all types of medications used by patients [3]. New methods based on an integrative approach have been developed over the past decade to decipher disease complexity and might be particularly relevant in the context of asthma [8]. Network models have been recently proposed in two lung diseases, i.e. COPD and asthma-COPD overlap (ACO), to map multimorbidity interrelationships [9, 10]. To the best of our knowledge, no study has used drug administrative databases to look for networks of multimorbidity-related medications. Moreover, to what extent multimedication influences asthma features, prognosis and therapeutic management is little known [6].

Asthma in the elderly is a phenotype of interest, especially in women because asthma tends to be more prevalent and more severe in women than in men [11]. Asthma research needs to account for the large endo-phenotypic heterogeneity to improve our understanding of the disease [12]. Clustering methods, which are able to account for multiple aspects of the disease, have identified clinically meaningful asthma phenotypes [13]. However, until now these approaches have focused on personal and clinical characteristics, and did not consider the large amount of information provided by drug administrative databases. We hypothesised that a pharmacoepidemiological study using clustering methods applied to drug administrative databases would provide a new insight into our understanding of asthma multimorbidity.

We aimed at comprehensively characterising medications used by elderly women with and without asthma, and assessing how multimedication impacts on asthma prognosis. Specific aims were: 1) to characterise multimedication and compare medication interrelationships among elderly women with and without asthma, 2) to comprehensively identify specific multimorbidity-related medication profiles in asthma, and 3) to prospectively assess the association between these profiles and asthma characteristics and subsequent adverse asthma events.

\section{Methods}

Population

The analysis was conducted on 17458 women participating in the Asthma-E3N study. Briefly, the Asthma-E3N study is a nested case-control study on asthma within the E3N study (Etude Epidémiologique auprès des femmes de la Mutuelle Générale de l'Education Nationale (MGEN)), a prospective study focused on major chronic diseases in female members of the MGEN, a French national health insurance plan covering mostly teachers $[14,15]$. The E3N study (11 successive questionnaires sent since 1990) and the Asthma-E3N study (2011-2012) provided detailed data on general features and respiratory health, respectively, complemented by a comprehensive outpatient dispensed medication database from 2004 onwards. Ever-asthma was defined by at least one positive answer to the question "Have you ever had an asthma attack?" as recommended by the American Thoracic Society Epidemiology Standardization Project, at least once between 1992 and 2008, and confirmed in the Asthma-E3N questionnaire either by a positive answer to that question or by the report of any asthma symptom [16].

The study protocol was approved by the French Institutional Ethics Committee and written informed consent was obtained for all participants. Ethical approval was granted to use the MGEN database.

\section{Estimation of medication exposure}

Non-hospital-delivered drug classes were identified over the 2 years before the Asthma-E3N questionnaire from the exhaustive MGEN drug database using the Anatomical Therapeutic Chemical (ATC) code as recommended by the World Health Organization and were grouped into categories [17]. Medication

This article has supplementary material available from erj.ersjournals.com

Received: Oct 132017 | Accepted after revision: March 012018 
exposure was defined within each category in three classes based on the average annual number of reimbursements: regular $(n \geqslant 3)$, intermittent $(1<n<3)$ and unexposed. Multimorbidity-related medication defined any medication apart from those for asthma.

\section{Asthma characteristics and adverse asthma events assessed at the Asthma-E3N study}

Current asthma was defined by the self-report of asthma symptoms or the use of asthma treatment over the previous 12 months. Late-onset asthma was defined as a disease onset (i.e. age at first asthma attack) after 40 years of age [18]. The term ACO was applied to women with self-report of both asthma and COPD. Asthma treatment level was assessed according to the Global Initiative for Asthma guidelines, and classified as no, low and high maintenance therapy [19].

Asthma control was assessed by the validated Asthma Control Test (ACT) over the previous month [20]. Women were classified into uncontrolled $($ ACT $\leqslant 19)$ and controlled asthma (ACT >19). Asthma attack was defined by a positive answer to "Have you had an asthma attack over the previous 12 months?" Asthma exacerbation was defined by the report of asthma attacks lasting at least 2 days in the previous 12 months. Health-related quality of life was assessed from the total score of the specific Asthma Quality of Life Questionnaire (AQLQ) [21].

Further details of asthma characteristics and adverse asthma events are given in the supplementary material.

\section{Sociodemographic and clinical variables}

Sociodemographic characteristics included age, body mass index (BMI; four classes: <20, 20-25, 25-30 and $\geqslant 30 \mathrm{~kg} \cdot \mathrm{m}^{-2}$ ), educational level (four classes: less than high school diploma, high school to level 2 university, level $3 / 4$ university and level $\geqslant 5$ university) and active smoking (three classes: never-smoker, ex-smoker and current smoker). The medical follow-up (frequency, medical speciality) and an asthma symptom score (defined as the sum of positive answers to five questions related to asthma symptoms and grouped into four classes: $0,1,2$ and $\geqslant 3$ ) were assessed over the 12 months that preceded the Asthma-E3N questionnaire. Parental history of asthma was categorised as none versus father and/or mother.

\section{Statistical analysis}

To address our first aim, we applied network-based analyses on all drug classes dispensed to women with and without asthma. We generated comprehensive network graphs using Gephi version 0.9.1 graph visualisation and manipulation software (https://gephi.org [22]), in which drug classes are represented by a node whose diameter is proportional to its prevalence and the connections between drug classes estimated by $\phi$ coefficients (i.e. Pearson's correlation coefficients for binary variables) are represented by the edges between nodes ( $p \leqslant 0.01$ as previously used in the literature) [23]. Analyses were performed in the same number of women with and without asthma to allow for a direct comparison of the two networks.

To address our second aim, we performed latent class analysis (LCA), i.e. a data-driven approach, to identify asthma groups characterised by specific multimorbidity-related medication profiles. The LCA was developed with 1) the 21 drug classes a priori linked to asthma-related multimorbidity and grouped into 10 categories (supplementary material), 2) drug classes linked with asthma medications in the network graph, and 3) three clinical and environmental factors that cannot be assessed by drug classes (BMI $\geqslant 30 \mathrm{~kg} \cdot \mathrm{m}^{-2}$ for obesity, sleep apnoea syndrome and smoking status).

To address our third aim, we prospectively assessed the association between each LCA-derived medication profile and asthma outcomes by regression models (crude and adjusted on age and asthma treatment), weighted by the posterior probability of belonging to the assigned cluster to account for the uncertainty in the cluster membership (see supplementary material). A sensitivity analysis was conducted in women with current asthma and in never-smokers with asthma to address the robustness of our findings.

Multiple imputation was performed to handle missing data derived from the Asthma-E3N questionnaire (see supplementary material). Analyses were performed using SAS version 9.4 (SAS Institute, Cary, NC, USA).

\section{Results}

\section{Population}

Among the 17458 women included in the Asthma-E3N study (mean (range) age 70.0 (61.3-87.5) years), $4328(24.8 \%)$ had ever-asthma, of whom 69.8\% ( $\mathrm{n}=3023)$ had current asthma (figure 1 and table 1). Women with ever-asthma were slightly younger ( $69.6 \pm 6.1$ versus $70.1 \pm 6.3$ years old; $\mathrm{p}<0.0001)$, more often obese $(9.9 \%$ versus $6.3 \%$; $\mathrm{p}<0.0001)$, more often never-/ex-smokers $(96.1 \%$ versus $95.3 \%$; $\mathrm{p}=0.02)$ and had 
a higher educational level (level $\geqslant 3$ university $40.0 \%$ versus $36.0 \%$; $<<0.0001$ ) than women without asthma. Most of the women used at least one nonasthma medication $(94.4 \%$ and $94.2 \%$ among women with and without asthma, respectively).

\section{Multimedication network-based analysis}

The number and types of drug classes were similar in women with and without asthma (177 versus 171, respectively) (supplementary table E1). However, the networks showed that the number of links between drug classes was higher in asthma, meaning that drugs from different classes were more often jointly delivered in women with asthma than in those without asthma (282 versus 160 statistically significant links and 251 versus 158 after excluding asthma drug classes) (figure 2). Among women without asthma (figure $2 \mathrm{a})$, the main reimbursed drug classes (defined by the ATC code) were other analgesics and antipyretics $(68.5 \%)$, and anti-inflammatory and antirheumatic products $(60.6 \%)$. The correlations varied from 0.10 to 0.47 (median (interquartile range (IQR)) $\phi=0.18(0.14-0.23)$ ) among the 160 links between drug classes identified by the network analysis.

Among women with ever-asthma (figure 2b), the main reimbursed drug classes were other analgesics and antipyretics $(72.2 \%)$, anti-inflammatory and antirheumatic products $(63.6 \%)$, antihistamines for systemic use (52.7\%), drugs for peptic ulcer and GORD (51.5\%), viral vaccines (50.2\%), and adrenergic inhalants (50.1\%). Among the 282 statistically significant links found between drug classes (median (IQR) $\phi=0.16$ $(0.13-0.21)), 31$ links associated asthma-related drug classes with other drug classes (median (IQR) $\phi=0.16$ (0.14-0.21)), including corticosteroids for systemic use $(\phi=0.29)$, anti-infective drugs $(\phi=0.28)$, antihistamines for systemic use $(\phi=0.27)$, topical decongestants $(\phi=0.27)$, drugs for peptic ulcer and GORD $(\phi=0.18)$, analgesics ( $\phi=0.14)$, anxiolytics $(\phi=0.14)$, drugs for bowel diseases $(\phi=0.12)$, and anti-inflammatory and antirheumatic products $(\phi=0.10)$. The number of drug classes and links between them were higher in women with current asthma than in those with past asthma (supplementary figure E1).

\section{Multimorbidity-related medication profiles in asthma}

The LCA model applied to the drug administrative data and personal characteristics identified three multimorbidity-related medication profiles (figure 3). The mean $\pm \mathrm{SD}$ of the maximum posterior probabilities of belonging to the assigned cluster was high $(0.84 \pm 0.17)$, indicating a high degree of certainty in the cluster membership.

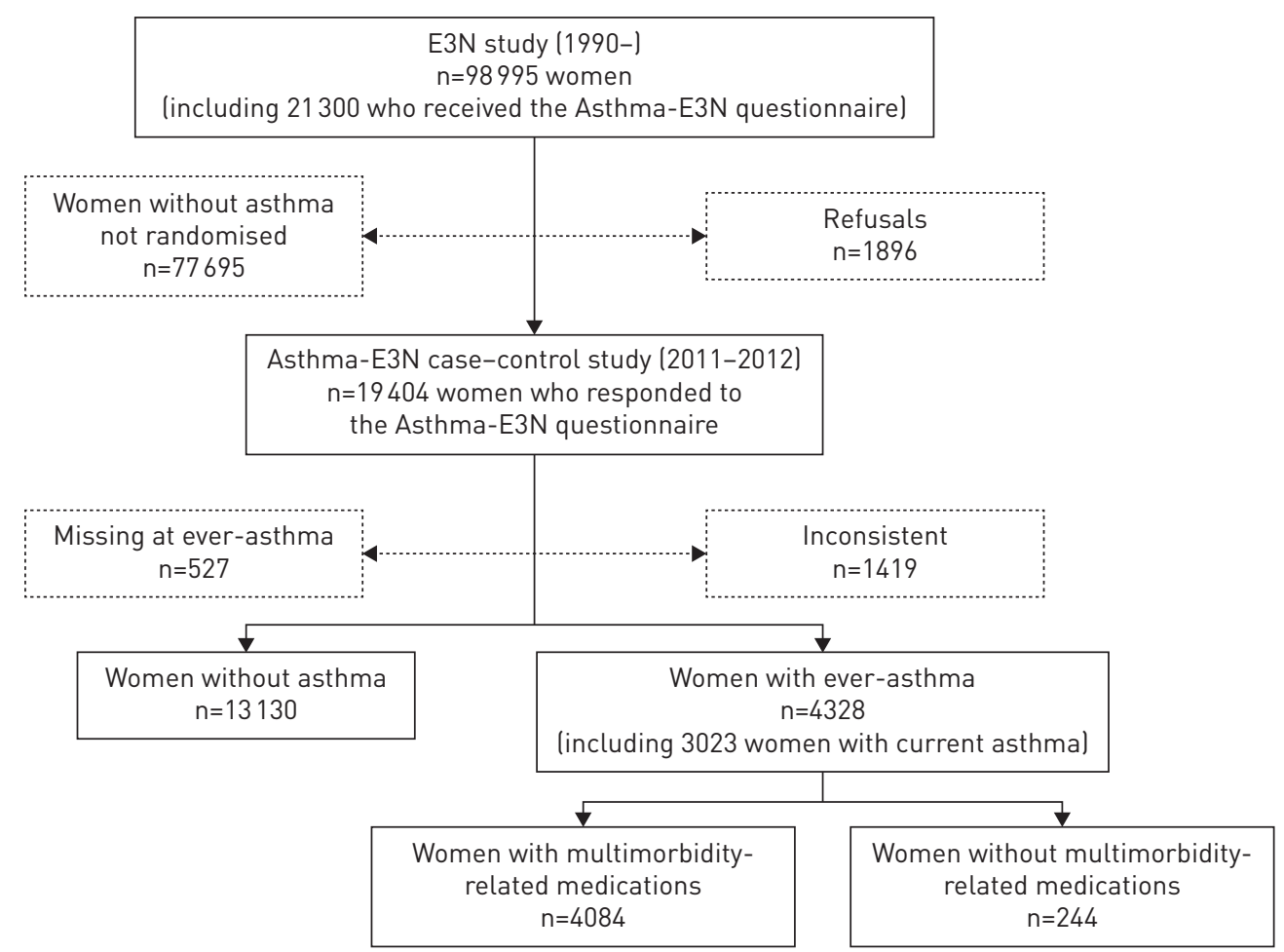

FIGURE 1 Flowchart of the study population. 
TABLE 1 Characteristics of women with ever-asthma from the Asthma-E3N study according to disease activity and the use of nonasthma medications

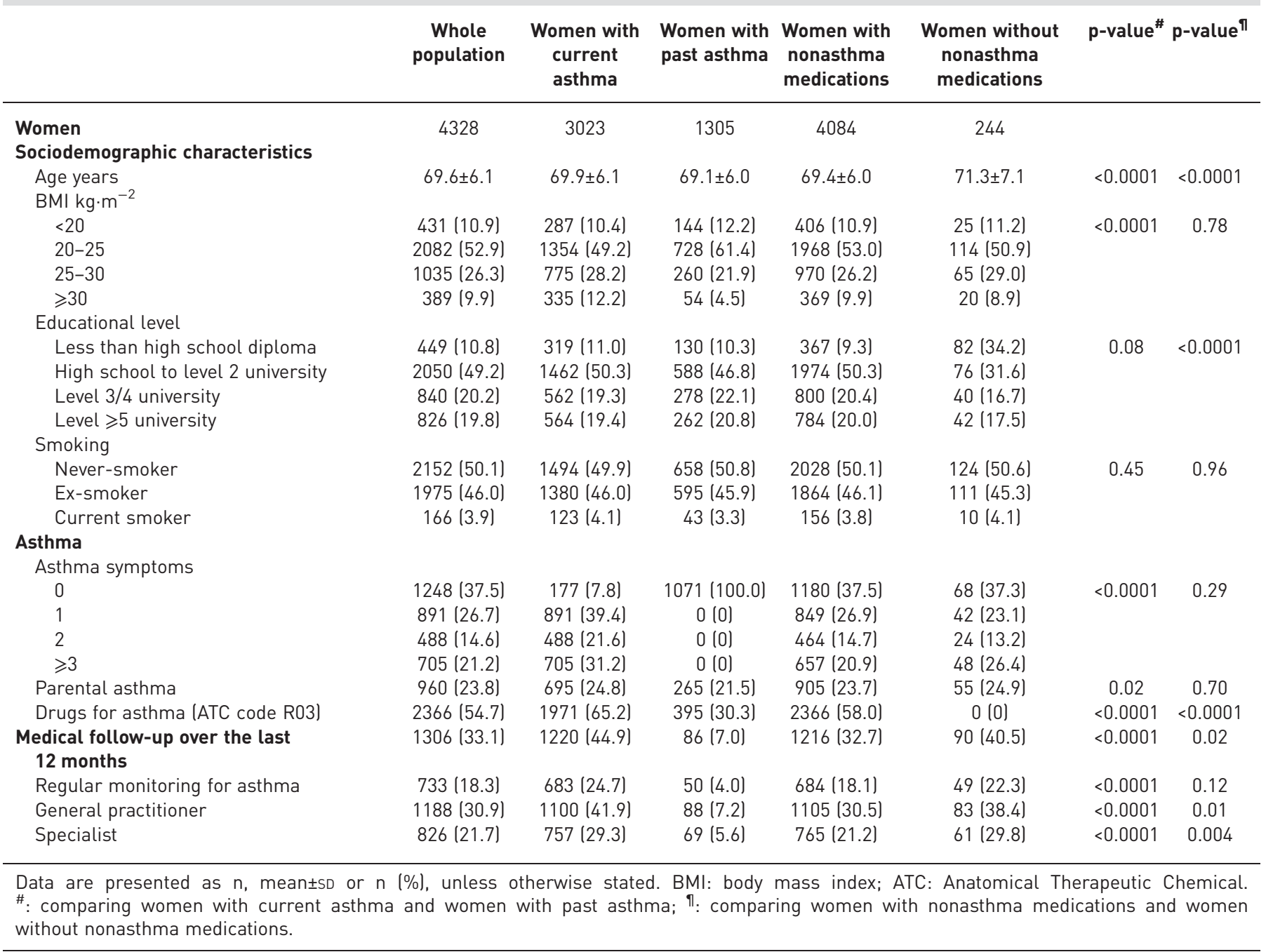

A first profile, characterised by few medications, was labelled "Few multimorbidity-related medications" $(\mathrm{n}=1885(43.5 \%))$. A second profile, mainly characterised by medications for allergy, nose diseases, respiratory diseases, rheumatism/inflammation, pain, GORD, hormonal disorders and anti-infective drugs, was labelled "Predominantly allergic multimorbidity-related medications" ( $\mathrm{n}=1419$ (32.8\%)). The third profile, mainly characterised by obesity, sleep apnoea syndrome, and medications for respiratory diseases, GORD, rheumatism/inflammation, pain, bowel diseases, psychological disorders, cardiovascular diseases, diabetes and dyslipidaemia, was labelled "Predominantly metabolic multimorbidity-related medications" $(\mathrm{n}=1024(23.7 \%)$ ) (figure 3 and supplementary figure E2). The analysis restricted to never-smokers $(\mathrm{n}=2152)$ showed similar profiles $(37.8 \%, 36.8 \%$ and $25.4 \%$, respectively).

The profiles differed in terms of individual and asthma characteristics. Compared with the two other profiles, women belonging to the "Predominantly metabolic multimorbidity-related medications" profile were older and had a lower educational level (table 2). Women belonging to the "Predominantly allergic multimorbidity-related medications" and "Predominantly metabolic multimorbidity-related medications" profiles more often had current asthma (table 2) and asthma maintenance therapy (OR 8.24, 95\% CI 6.809.99 and 4.12 , 95\% CI 3.33-5.10, respectively) than those with few multimorbidity-related medications. They also more often had late-onset asthma (OR 1.36, 95\% CI 1.12-1.64 and 1.67, 95\% CI 1.40-2.00, respectively) and ACO (OR 1.98, 95\% CI 1.54-2.53 and 1.91, 95\% CI 1.43-2.54, respectively).

Multimorbidity-related medication profiles and subsequent adverse asthma events

Compared with women belonging to the "Few multimorbidity-related medications" profile (reference group), women belonging to the two other profiles had more often uncontrolled asthma, asthma attacks, 


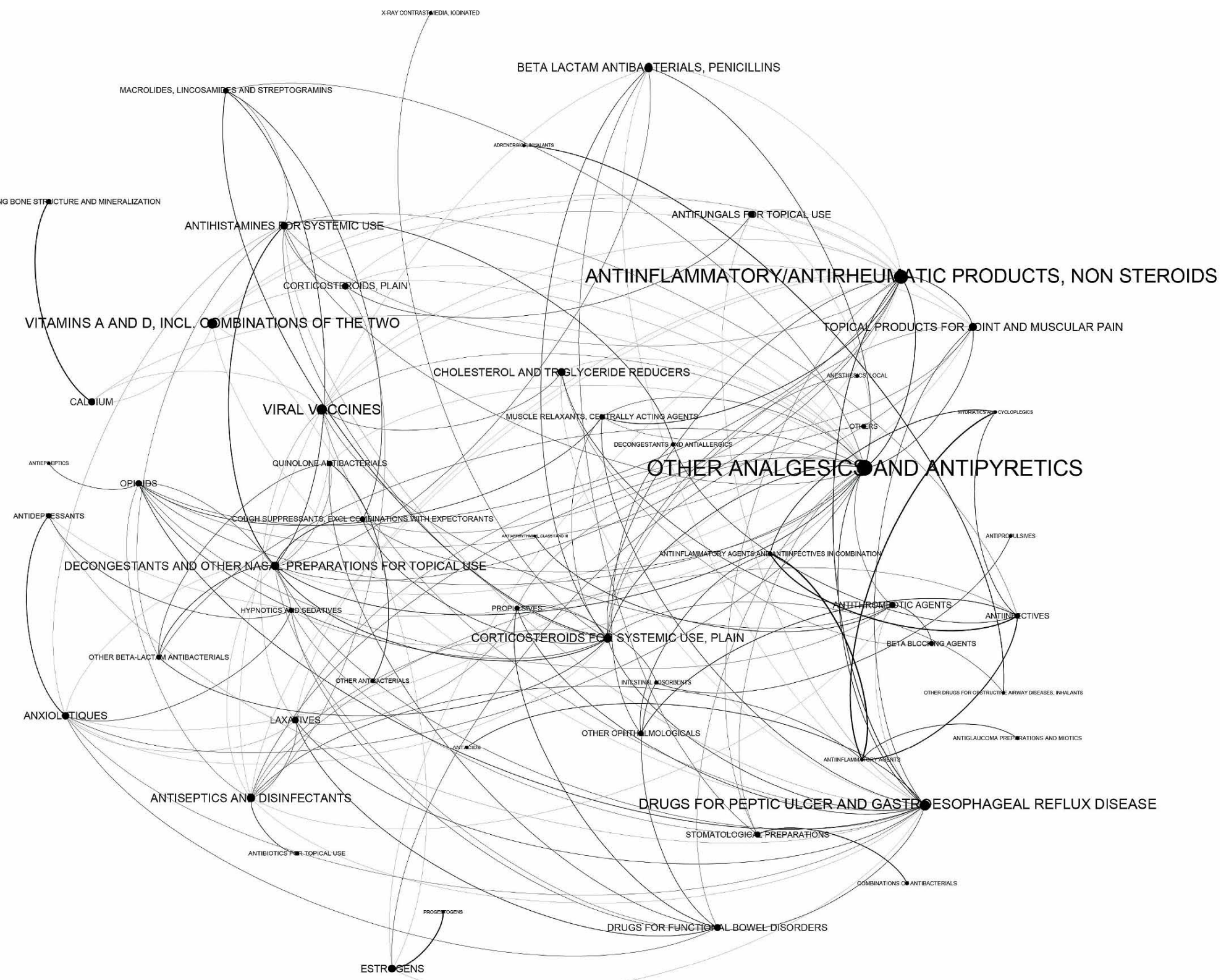

FIGURE 2 Network-based analyses among a) 4328 women without asthma randomly selected and b) the 4328 elderly women with ever-asthma from the Asthma-E3N study. Network graphs were generated using Gephi (https://gephi.org [22]] and were built from all drug classes according to the third level of the Anatomical Therapeutic Chemical classification. Node diameters represent the prevalence of use of each drug class in the population. Edge thicknesses represent the strength of the association between two drug classes estimated by the $\phi$ coefficient. In (b), green indicates asthma drug classes and statistically significant links between them; orange indicates other relevant drug classes and statistically significant links between them and 
MACROLIDES, LINCOSAMIDFSAND STREPTOGRAMINS<smiles>CC1CC[C@H]1CO</smiles>

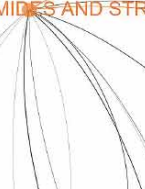

(n)

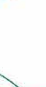

ADRENERGICS, INHALANTS

ANTIHISTAMINES SPR SVSTENYLUSE

VITAMINS A AND D, INCL. COMBINATIONS OF THE TWO
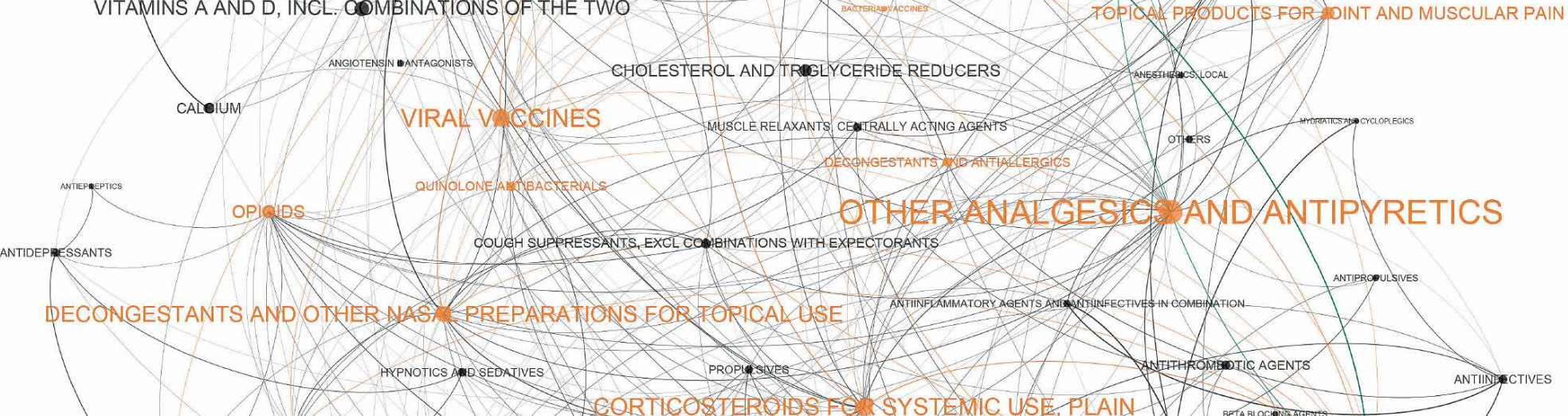

OTHERGETA-LACTIMANTBGACTERIALS

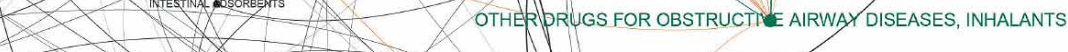

ANXIOLULLUES LAXHINES

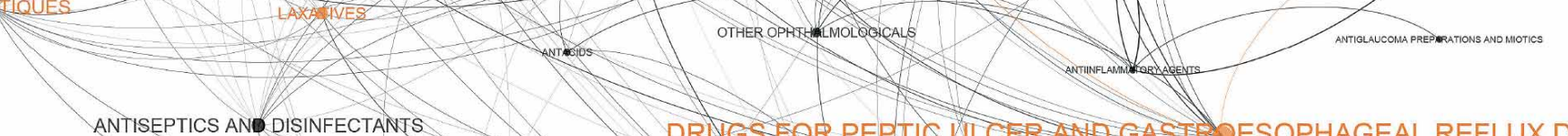

ANTISEPTICS AND DISINFECTANTS

DREGSTRR PEPTIG UILCERIANB_GASISDESOPHAGEAL REFLUX DISEASE

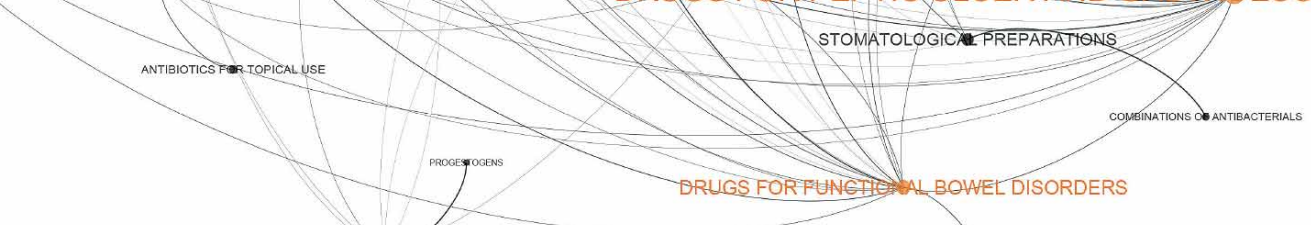

ESTROGENS 


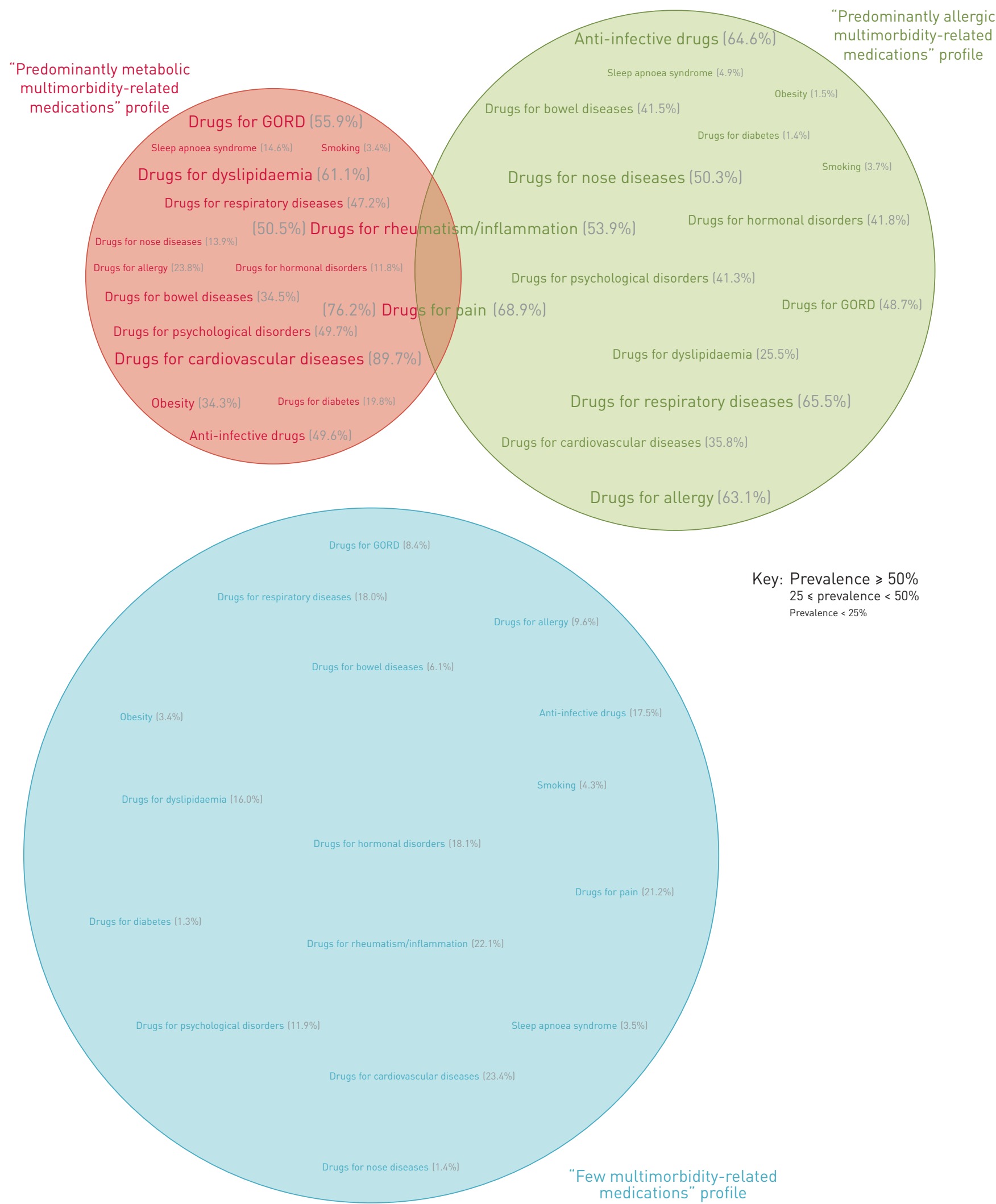

FIGURE 3 Multimorbidity-related medication profiles identified by latent class analysis: "Few multimorbidity-related medications" profile (n=1885 (43.5\%)), "Predominantly allergic multimorbidity-related medications" profile ( $\mathrm{n}=1419$ (32.8\%)) and "Predominantly metabolic multimorbidity-related medications" profile ( $n=1024$ (23.7\%)). GORD: gastro-oesophageal reflux disease. Overlaps between profiles were considered when at least $50 \%$ of women belonging to each profile had at least one reimbursement of a drug class during the study period. 
TABLE 2 Characteristics of women belonging to the three multimorbidity-related medication profiles identified by latent class analysis

$\begin{array}{ccc}\text { Whole } & \text { “Few } & \text { “Predominantly allergic } \\ \text { population } & \begin{array}{c}\text { multimorbidity-related } \\ \text { medications" profile }\end{array} & \begin{array}{c}\text { multimorbidity-related } \\ \text { medications" profile }\end{array}\end{array}$

medications" profile

\author{
“Predominantly p-value \\ metabolic \\ multimorbidity-related \\ medications" profile
}

\begin{tabular}{|c|c|c|c|c|c|}
\hline Women & 4328 & 1885 & 1419 & 1024 & \\
\hline \multicolumn{6}{|l|}{ Sociodemographic characteristics } \\
\hline Age years & $69.6 \pm 6.1$ & $68.9 \pm 5.9$ & $69.3 \pm 5.8$ & $71.6 \pm 6.5$ & $<0.0001$ \\
\hline \multicolumn{6}{|l|}{ Educational level } \\
\hline Less than high school diploma & $449(10.8)$ & $185(10.1)$ & $118(8.7)$ & $146(14.9)$ & \multirow[t]{4}{*}{$<0.0001$} \\
\hline High school to level 2 university & $2050(49.2)$ & $822(45.1)$ & 700 (51.5) & 528 (53.8) & \\
\hline Level 3/4 university & $840(20.2)$ & $416(22.8)$ & $271(19.9)$ & $153(15.6)$ & \\
\hline Level $\geqslant 5$ university & $826(19.8)$ & $402(22.0)$ & $270(19.9)$ & $154(15.7)$ & \\
\hline \multicolumn{6}{|l|}{ Asthma therapeutic management } \\
\hline High level of asthma maintenance therapy & $632(14.6)$ & $95(5.0)$ & $356(25.1)$ & $181(17.7)$ & \multirow[t]{3}{*}{$<0.0001$} \\
\hline Low level of asthma maintenance therapy & $620(14.3)$ & $133(7.1)$ & $327(23.0)$ & $160(15.6)$ & \\
\hline No asthma maintenance therapy & $3076(71.1)$ & 1657 (87.9) & 736 (51.9) & $683(66.7)$ & \\
\hline \multicolumn{6}{|l|}{ Asthma characteristics } \\
\hline Current asthma & 3023 (79.9) & 1119 (70.9) & 1098 (85.8) & 806 (87.3) & $<0.0001$ \\
\hline Late-onset asthma & 1455 (36.7) & 545 (31.5) & $517(39.0)$ & 393 (43.3) & $<0.0001$ \\
\hline Asthma-COPD overlap & 863 (22.7) & 265 (15.9) & 353 (28.3) & $245(27.5)$ & $<0.0001$ \\
\hline \multicolumn{6}{|l|}{ Adverse asthma events } \\
\hline Uncontrolled asthma & $522(19.2)$ & $127(12.2)$ & 214 (21.5) & $181(26.7)$ & $<0.0001$ \\
\hline At least one asthma attack & 1055 (25.4) & $317(17.5)$ & $438(32.3)$ & $300(30.6)$ & $<0.0001$ \\
\hline At least one asthma exacerbation & 446 (13.2) & 123 (8.8) & 206 (17.9) & $117(14.3)$ & $<0.0001$ \\
\hline Total AQLQ score & $6.0 \pm 1.0$ & $6.3 \pm 0.9$ & $5.9 \pm 1.0$ & $5.8 \pm 1.1$ & $<0.0001$ \\
\hline
\end{tabular}

asthma exacerbations (table 2 and figure 4) and lower AQLQ score $(\beta \pm$ SD $5.94 \pm 0.03 ; \mathrm{p}<0.0001$ and 5.86 $\pm 0.03 ; \mathrm{p}<0.0001$ for the "Predominantly allergic multimorbidity-related medications" and "Predominantly metabolic multimorbidity-related medications" profiles, respectively). Associations remained similar in the models adjusted for age or for asthma treatment level and when restricting the analysis to women with current asthma (supplementary tables E2 and E3). The associations among never-smokers showed patterns similar to those observed in the whole population, although some associations were no longer statistically significant due to decreased sample size (supplementary table E4).

\section{Discussion}

To the best of our knowledge, this is the first pharmacoepidemiological study using an unbiased approach relying on drug administrative databases to characterise asthma-related multimorbidity. Nonasthma drugs were more often jointly delivered in women with asthma than in those without asthma, indicating that multimorbidity is more common in asthma. We identified two multimorbidity medication profiles, one mainly characterised by predominantly allergic multimorbidity and the other by predominantly metabolic multimorbidity, which were prospectively associated with subsequent adverse asthma events.

The strength of this study relates to the population. First, this study focused on the elderly, a population of particular interest in terms of multimorbidity and thus of multimedication. More than half of the population aged $\geqslant 65$ years suffers from multimorbidity (i.e. at least two chronic diseases), leading to a five times increase in healthcare expenditure compared with nonmultimorbid people [24]. Our findings relying on a large population of elderly women are of public health relevance because of higher asthma prevalence and severity in elderly women than in elderly men [11]. However, studies in elderly men with asthma are needed to address the generalisability of our findings. Indeed, although there is no clear evidence for sex differences in the rate of multimorbidity in asthma [25], the distribution of the multimorbidity medication profiles might differ between sexes because of differences in disease perception, in hormonal, environmental and occupational factors, and in adherence to medications between men and women with asthma [26]. Second, the population focused on women with ever-asthma covers a wide range of asthma phenotypes, and therefore allows addressing the role of disease activity and severity in multimorbidity. Although asthma was defined through a repeated answer to a standardised questionnaire widely used in 
a)

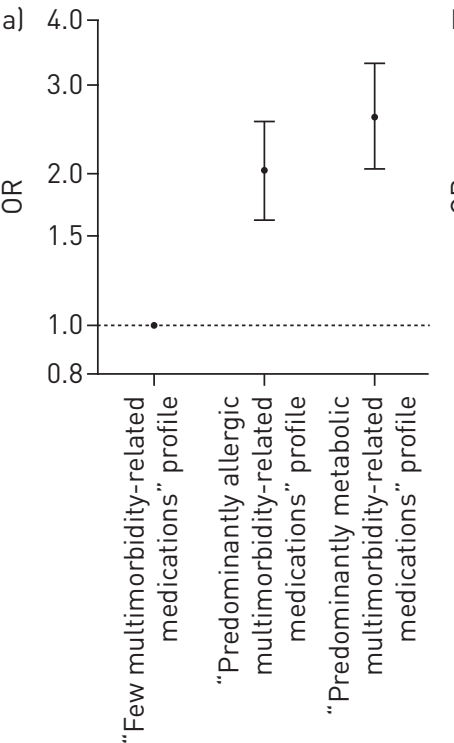

b)

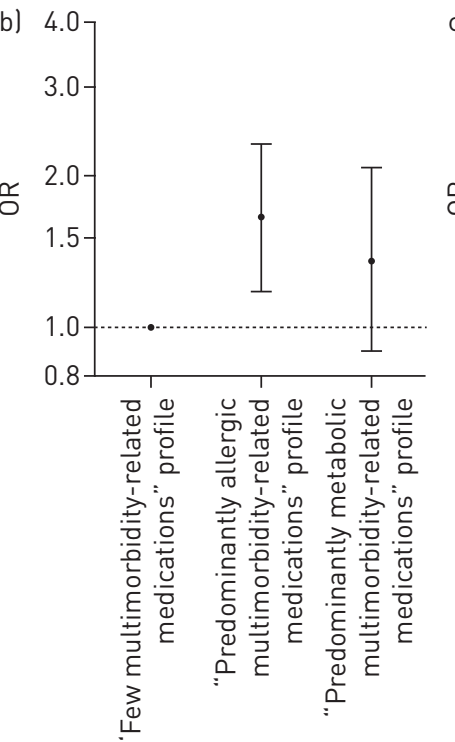

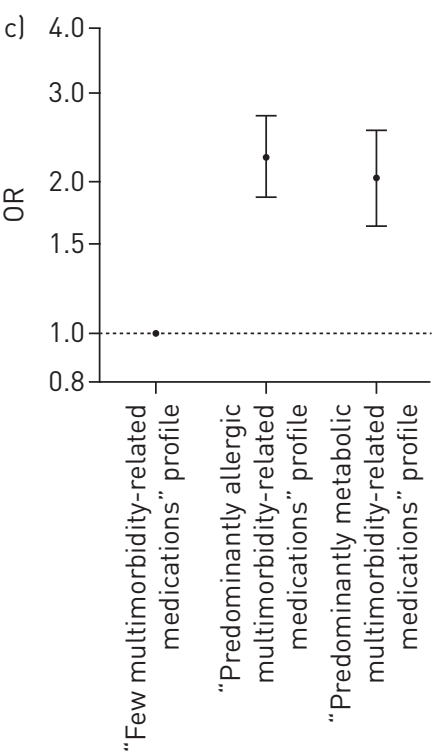

FIGURE 4 Associations lodds ratio and 95\% confidence interval) between multimorbidity-related medication profiles identified by latent class analysis and adverse asthma events: a) uncontrolled asthma, b) asthma exacerbations and c) asthma attacks.

epidemiological studies [16, 27], misdiagnosis remains questionable in this elderly population. However, the sensitivity analysis restricted to never-smokers showed similar patterns of associations, suggesting the robustness of our findings to potential asthma-COPD misdiagnosis. Finally, the detailed individual and phenotypic characteristics provided by the Asthma-E3N questionnaire offer the unique opportunity to estimate the clinical significance of the identified multimedication profiles.

A further strength of this study lies on the use of a comprehensive and exhaustive dispensed drug database allowing multimedication network-based analysis among elderly women. Already used in several countries for drug surveillance, administrative databases are a unique and powerful source of data to estimate drug use in real life in large populations [28]. Our study expands the opportunity of using such databases by demonstrating their relevance in identifying groups of individuals characterised by their medication profiles. We acknowledge that drug administrative databases are a proxy for medication use and the assessment of medication exposure may be hampered by nonadherence, which is commonly encountered in chronic diseases [29]. This weakness is difficult to avoid, but is probably minimised in this study conducted on women with a higher mean educational level than the general population [29]. A further limitation in our approach relies on the postulate that medications are a substitute for the diagnosis made by a physician; dispensed drug databases might not accurately assess diseases in some contexts. However, the ATC classification used in our study is a standardised and validated classification according to the main therapeutic indication of medications, recommended and promoted by the World Health Organization [17].

We provide here the relevance of the pharmacoepidemiological approach to decipher asthma complexity and heterogeneity. Most studies suffer from a reductionist approach considering only a limited number of diseases, therefore missing the overall picture [9]. Only few studies have considered lung diseases as an integrative system [8-10] and none by using a pharmacoepidemiological approach [30]. The multimedication network-based analysis offers an integrative approach to understand complex diseases such as asthma. Therefore, we added a new layer to the previous networks by providing a comprehensive overview of the therapeutic management of individuals with chronic diseases, which we called the "pharmacome" in reference to the "-ome" family. An important challenge now is to establish the relationships between the different levels of networks (i.e. genome, proteome, metabolome, phenome, pharmacome and exposome).

Our findings confirm that multimedication is common in ambulatory care in elderly people and particularly in those with asthma [18]. We found interconnections between asthma medications and the main drug classes used for known asthma comorbidities. However, we did not find all expected connections, especially for drugs for cardiovascular diseases, probably because of their wide range of use and the rather high socioeconomic status of our population of elderly women [31]. Interestingly, our study supports the relationship between asthma and rheumatism/inflammation, which might have some 
implications in the management of these patients by considering leukotriene receptor antagonists as a therapeutic option [32].

Our unsupervised approach identified three clinically relevant profiles of elderly women with asthma characterised by multimedication. In our highly educated population, a large proportion of elderly women (43.5\%) had few multimorbidity-related medications, a characteristic of healthy ageing that is influenced by a number of factors including demographics, functional status, health risks, social context and resources [33]. About a quarter of our elderly population belonged to the "Predominantly allergic multimorbidity-related medications" profile. This frequency is much lower than in studies relying on objective measurements of allergic sensitisation in younger adults, where about two-thirds of adults with ever-asthma had allergic-related phenotypes [34]. This profile relates to the allergic multimorbid phenotype, clearly identified in children, but still poorly studied later in life. It is characterised by a high use of drugs for hormonal disorders, supporting the relationship between hormonal effects and allergic diseases [35]. The last profile associated with late-onset asthma identified women with a high proportion of metabolic multimorbidity-related medications. This observation is in agreement with a recent study indicating that later age at asthma onset predicts the long-term risk of cardiovascular diseases, particularly in women [36]. The two profiles characterised by multimedication were associated with a higher level of asthma treatment, suggesting that multimorbidity is associated with disease severity.

Consistent with the literature indicating that multiple comorbidities may hamper asthma control [37], we showed that the two clusters characterised by multimedication had a higher risk of subsequent adverse asthma events. The highest risk of asthma exacerbation and asthma attack was observed in the "Predominantly allergic multimorbidity-related medications" profile; this finding extends to the elderly the previous observations in children showing an association between the allergic multimorbid phenotype and asthma severity [38]. The association between uncontrolled asthma and the "Predominantly metabolic multimorbidity-related medications" profile, to which obese patients mostly belonged, supports the increasing literature on the causal role of obesity on asthma [39]. This finding also contributes to the growing literature on the association between metabolic syndrome and lung diseases, including asthma, although the mechanisms behind this association remain to be elucidated [39]. Multimorbidity might impact asthma through different mechanisms, including involvement in similar pathophysiological pathways, in disease evolution or in modulating asthma phenotype, but none of these mechanisms have been extensively described $[5,6]$. In our study, the associations between multimorbidity and subsequent adverse asthma events were observed independently from disease severity, suggesting that factors other than asthma severity are involved in this association.

Our findings have relevant clinical implications. First, among elderly women with asthma, we identified a common subgroup of women with complex multimorbidities (allergic or metabolic), who most likely require a continuity of care by a physician and regular consultations to review their chronic conditions and related medications $[25,40]$. Second, we showed that half of the patients belonging to the predominantly metabolic multimorbidity profile used drugs for psychological disorders. This novel finding emphasises that asthma management of these multimorbid patients should consider both physical and mental health dimensions [25]. Third, our results reinforce that the "current one-size-fits-all" therapeutic management in asthma has to be reconsidered towards personalised medicine integrating the multimorbidity dimension [40]. From a wider point of view, the current management approach considering single-disease clinical guidelines may not be applicable to patients with multimorbidity, partly because these patients are routinely excluded from clinical trials on which the guidelines rely [2]. A broader approach with guidelines that consider clusters of chronic conditions might be a step forward [40]. Such an approach is particularly relevant given population ageing.

In conclusion, using the "pharmacome" as an integrative approach, we showed that multimedication, often encountered in the elderly with asthma, predicts poor asthma prognosis. Among the factors that contribute to the unmet needs in asthma management, multimorbidity should particularly be considered. A multifaceted strategy is needed for asthma patients with multimorbidity to successfully restore and maintain the control of the disease, which should ultimately have a substantial medicoeconomic impact.

Acknowledgements: The authors would like to thank Marie Fangon, Maryvonne Niravong and Lyan Hoang (INSERM, Centre for Research in Epidemiology and Population Health (CESP), Health Across Generation Team, Villejuif, France) for the implementation of the study. They are indebted to all E3N participants, without whom this study would not have been possible for the quality of their involvement in the E3N study.

Support statement: This work was supported by a grant from the Institut pour la Recherche en Santé Publique (IReSP), and the joint support of Direction Générale de la Santé (DGS), Mission recherche de la Direction de la recherche, des études, de l'évaluation et des statistiques (Mire-DREES), Caisse nationale d'assurance maladie des travailleurs salariés (CNAMTS), Régime Social des Indépendants (RSI) and Caisse nationale de solidarité pour l'autonomie (CNSA). In 
addition, the work was supported by a state grant ANR-10-COHO-0006 from the Agence Nationale de la Recherche within the Programme des Investissements d'Avenir. The research was performed using data from the INSERM E3N cohort and support from the Mutuelle Générale de l'Education Nationale (MGEN), the Gustave Roussy Institute (IGR), the French League against Cancer (LNCC), and the National Institute for Health and Medical Research (INSERM) for setting up and maintaining the cohort. Funding information for this article has been deposited with the Crossref Funder Registry.

Conflict of interest: S. Chanoine reports personal fees (board membership) from AstraZeneca, and nonfinancial support (travel/accommodation/meeting expenses) from Boehringer Ingelheim, Actelion Pharmaceuticals and MSD, outside the submitted work. I. Pin reports travel grants and presentation fees from Novartis and AstraZeneca, and fees for training courses from Vertex, outside the submitted work. C. Pison has received grants and lectures fees from GlaxoSmithKline, AstraZeneca, Novartis and BIF, as well as speaker fees and fees for meeting participation from GlaxoSmithKline, AstraZeneca, Novartis, BIF and Teva. J. Bousquet reports personal fees (scientific and advisory boards) from Almirall, Meda, Merck, MSD, Novartis, Sanofi-Aventis, Takeda, Teva and Uriach, as well as personal fees (lecture fees) from Almirall, AstraZeneca, Chiesi, GlaxoSmithKline, Meda, Menarini, Merck, MSD, Novartis, Sanofi-Aventis, Takeda, Teva and Uriach, outside the submitted work. P. Bedouch reports fees for meeting expenses from Actelion Pharmaceuticals, outside the submitted work. R. Varraso reports grants from the Institut pour la Recherche en Santé Publique (IReSP), during the conduct of the study. V. Siroux reports personal fees (speakers honorarium) from Teva, AstraZeneca and Novartis, outside the submitted work.

\section{References}

$1 \quad$ Gibson PG, McDonald VM, Marks GB. Asthma in older adults. Lancet 2010; 376: 803-813.

2 Farmer C, Fenu E, O'Flynn N, et al. Clinical assessment and management of multimorbidity: summary of NICE guidance. BMJ 2016; 354: i4843.

3 Song WJ, Cho SH. Challenges in the management of asthma in the elderly. Allergy Asthma Immunol Res 2015; 7: 431-439.

4 Weatherburn CJ, Guthrie B, Mercer SW, et al. Comorbidities in adults with asthma: population-based cross-sectional analysis of 1.4 million adults in Scotland. Clin Exp Allergy 2017; 10: 1246-1252.

$5 \quad$ Boulet LP. Influence of comorbid conditions on asthma. Eur Respir J 2009; 33: 897-906.

6 Kankaanranta H, Kauppi P, Tuomisto LE, et al. Emerging comorbidities in adult asthma: risks, clinical associations, and mechanisms. Mediators Inflamm 2016; 2016: 3690628

7 Gershon AS, Wang C, Guan J, et al. Burden of comorbidity in individuals with asthma. Thorax 2010; 65: 612-618.

8 Faner R, Cruz T, López-Giraldo A, et al. Network medicine, multimorbidity and the lung in the elderly. Eur Respir J 2014; 44: 775-788.

9 Divo MJ, Casanova C, Marin JM, et al. COPD comorbidities network. Eur Respir J 2015; 46: 640-650.

10 van Boven JF, Román-Rodríguez M, Palmer JF, et al. Comorbidome, pattern, and impact of asthma-COPD overlap syndrome in real life. Chest 2016; 149: 1011-1020.

11 Reed CE. Asthma in the elderly: diagnosis and management. J Allergy Clin Immunol 2010; 126: 681-687.

12 Gauthier M, Ray A, Wenzel SE. Evolving concepts of asthma. Am J Respir Crit Care Med 2015; 192: 660-668.

13 Siroux V, Basagaña X, Boudier A, et al. Identifying adult asthma phenotypes using a clustering approach. Eur Respir J 2011; 38: 310-317.

14 Clavel-Chapelon F, van Liere MJ, Giubout C, et al. E3N, a French cohort study on cancer risk factors. E3N Group. Etude Epidémiologique auprès de femmes de l'Education Nationale. Eur J Cancer Prev 1997; 6: 473-478.

15 Sanchez M, Varraso R, Bousquet J, et al. Perceived 10-year change in respiratory health: reliability and predictive ability. Respir Med 2015; 109: 188-199.

16 Ferris BG. Epidemiology Standardization Project (American Thoracic Society). Am Rev Respir Dis 1978; 118: 1-120.

17 WHO Collaborating Centre for Drug Statistics Methodology. Guidelines for ATC classification and DDD assignment. 2015. www.whocc.no/news/guidelines_for_atc_classification_and_ddd_assignment Date last accessed: June 13, 2017.

18 Yáñez A, Cho SH, Soriano JB, et al. Asthma in the elderly: what we know and what we have yet to know. World Allergy Organ J 2014; 7: 8.

19 Global Initiative for Asthma. Global Strategy for Asthma Management and Prevention. 2017. http://ginasthma.org/ 2017-gina-report-global-strategy-for-asthma-management-and-prevention Date last accessed: June 13, 2017.

20 Nathan RA, Sorkness CA, Kosinski M, et al. Development of the asthma control test: a survey for assessing asthma control. J Allergy Clin Immunol 2004; 113: 59-65.

21 Juniper EF, Guyatt GH, Ferrie PJ, et al. Measuring quality of life in asthma. Am Rev Respir Dis 1993; 147: 832-838.

22 Bastian M, Heymann S, Jacomy M. Gephi: an open source software for exploring and manipulating networks. 2009. https://gephi.org/publications/gephi-bastian-feb09.pdf Date last accessed: June 13, 2017.

23 Hidalgo CA, Blumm N, Barabási AL, et al. A dynamic network approach for the study of human phenotypes. PLoS Comput Biol 2009; 5: e1000353.

24 Bähler C, Huber CA, Brüngger B, et al. Multimorbidity, health care utilization and costs in an elderly community-dwelling population: a claims data based observational study. BMC Health Serv Res 2015; 15: 23.

25 Morrison D, Agur K, Mercer S, et al. Managing multimorbidity in primary care in patients with chronic respiratory conditions. NPJ Prim Care Respir Med 2016; 26: 16043.

26 Zein JG, Erzurum SC. Asthma is different in women. Curr Allergy Asthma Rep 2015; 15: 28

27 Sanchez M, Bousquet J, Le Moual N, et al. Temporal asthma patterns using repeated questionnaires over 13 years in a large French cohort of women. PLoS One 2013; 8: e65090.

28 Takahashi Y, Nishida Y, Asai S. Utilization of health care databases for pharmacoepidemiology. Eur J Clin Pharmacol 2012; 68: 123-129.

29 Dima AL, Hernandez G, Cunillera O, et al. Asthma inhaler adherence determinants in adults: systematic review of observational data. Eur Respir J 2015; 45: 994-1018. 
30 Hu JX, Thomas CE, Brunak S. Network biology concepts in complex disease comorbidities. Nat Rev Genet 2016; 17: 615-629.

31 Santos AC, Ebrahim S, Barros H. Gender, socio-economic status and metabolic syndrome in middle-aged and old adults. BMC Public Health 2008; 8: 62.

32 Rolfes MC, Juhn YJ, Wi CI, et al. Asthma and the risk of rheumatoid arthritis: an insight into the heterogeneity and phenotypes of asthma. Tuberc Respir Dis 2017; 80: 113-135.

33 Young HM, Cochrane BB. Healthy aging for older women. Nurs Clin North Am 2004; 39: 131-143.

34 Siroux V, González JR, Bouzigon E, et al. Genetic heterogeneity of asthma phenotypes identified by a clustering approach. Eur Respir J 2014; 43: 439-452.

35 Bonds RS, Midoro-Horiuti T. Estrogen effects in allergy and asthma. Curr Opin Allergy Clin Immunol 2013; 13: 92-99.

36 Tattersall $\mathrm{MC}$, Barnet $\mathrm{JH}$, Korcarz CE, et al. Late-onset asthma predicts cardiovascular disease events: the Wisconsin Sleep Cohort. I Am Heart Assoc 2016; 5: e003448.

37 Hekking PW, Amelink M, Wener RR, et al. Comorbidities in difficult-to-control asthma. J Allergy Clin Immunol Pract 2017; 6: 108-113.

38 Bousquet J, Anto JM, Just J, et al. The multimorbid polysensitized phenotype is associated with the severity of allergic diseases. J Allergy Clin Immunol 2017; 139: 1407-1408.

39 Baffi CW, Wood L, Winnica D, et al. Metabolic syndrome and the lung. Chest 2016; 149: 1525-1534.

40 Wallace E, Salisbury C, Guthrie B, et al. Managing patients with multimorbidity in primary care. BMJ 2015; 350 : h176. 